\title{
The Necessity of Human Kidney and Artificial Kidneys for the Human Health
}

Pati $\mathbf{P}^{1^{\star}}$ and Rathore $\mathbf{S K}^{2}$

${ }^{1}$ District Microbiologist, National Health Mission, Odisha, India

${ }^{2}$ Department of Zoology, Khallikote College, Odisha, India

"Corresponding author: Pallabi Pati, District Microbiologist, National Health Mission, Odisha, India, Tel: 0674 2392480; E-mail: ricky_pati@yahoo.co.in

Received date: Mar 18, 2018; Accepted date: Apr 07, 2018; Published date: Apr 12, 2018

Copyright: ( 2018 Pati P, et al. This is an open-access article distributed under the terms of the Creative Commons Attribution License, which permits unrestricted use, distribution, and reproduction in any medium, provided the original author and source are credited.

\begin{abstract}
The pair of kidney is one of the most important organs in human body playing many crucial roles in homeostasis, reabsorption, hormone production, filtration and removal of wastes from blood. The proper function of kidney is a basic requirement of human body but the partial or complete dysfunction of kidney result in several kinds of medical illness. The deposition of waste material due to non-filtration of blood is a major issue and dysbalance of homeostasis result into failure of the kidney. One of the temporary protocol termed as dialysis observed to be very helpful for the treatment and betterment during kidney failure. In dialysis, blood can be artificially filtered by means of a manmade machine which is a temporary artificial process of blood cleaning and filtration. This dialysis many times proves as a great life saver.
\end{abstract}

Keywords: Kidney; Homeostasis; Dialysis

\section{Introduction}

Kidneys are the main excretory organ basically located inside the posterior region of the abdominal cavity. Human kidneys are playing great role in the maintenance of the water, acid-base and electrolyte balance, thus it helps in the regulation of blood pressure. Kidneys can produce important hormones like erythropoietin, calcitriol and enzyme like renin. The most important function of the kidney is the filtration of blood from the body, which remove harmful waste materials along with excess amount of nutrients by means of urine through the urinary bladder. The excretory material termed as urine generally contains ammonia and urea. Kidneys also help in the reabsorption by which it again reabsorbs important amino acids, glucose and water. Reabsorption is performed by means of the nephron tubules which are originally present inside the kidney. During this process essential ion, molecules and nutrients which are required by the human body are again reabsorbed as the blood filtrate passing through the nephron tubules [1,2]. There are various kinds of kidney diseases that may include both nephrotic and nephritic syndrome such as acute kidney injury, chronic kidney disease, obstruction of urinary tract and renal cyst where the glomerular filtration rate is persistently hampered [3]. Such clinical condition of kidneys ultimately affects the basic function of kidneys and then there is an urgent need of a substitute which can help the body for its waste removal so that health status of the person is not compromised. So when the function kidney was in a declined state, dialysis treatment become very necessary for the removal of waste materials from the body otherwise the waste materials would be deposited in the blood and make it very toxic. Thus, the removal of waste material is necessary for the life expectancy. There are two kinds of dialysis process one is hemodialysis and another one is peritoneal dialysis process [3]. These processes are very much helpful for the survival of the affected patient and a great relief to the medical science as well as the health sector.

\section{The basic information about the structure and the function of human kidney}

Every human being has a pair of bean shaped kidneys (two in numbers) with a concave and convex surface. The kidneys are situated in the posterior region of the abdominal cavity placed in a retroperitoneal position having a little bit oblique angle, situated at both the right and left side of the vertebral columns, for which it is also known as the paired retroperitoneal organs. Both kidney's lower pole lies just opposite to the lumbar vertebral column number three and the upper pole lies in the opposite to the thoracic vertebral column number twelve. The right kidney is situated a bit more caudally than the left one. The left kidney is little bit larger than the right one. An adrenal gland also known as suprarenal gland is present on the top most region of kidney. Both the kidneys and the respective glands are surrounded by two layers such as pararenal and perirenal fat and a renal fascia, again the ribs numbered as eleventh and twelfth partially protects the uppermost portion of the kidney and the adrenal gland $[1,2]$. The weight of kidney ranges from $125 \mathrm{~g}$ to $170 \mathrm{~g}$ in case of adult male and whereas kidney weighs from $115 \mathrm{~g}$ to $155 \mathrm{~g}$ in case of adult female [4]. Both of the kidneys have a specific range of medial renal volumes where the median renal volume for the left kidney is $146 \mathrm{~cm}^{3}$ and for the right kidney is $134 \mathrm{~cm}^{3}$ [5].

\section{The basic components help in the function of human kidney}

The concave surface of both of the kidneys called as hilum through which renal artery enters inside the kidneys whereas the renal vein and ureter exit from the kidney. The kidneys receive approximately $20 \%$ of the cardiac output blood from the renal arteries which are two in numbers (paired arteries), and drained into the two renal veins (paired veins). The final waste after filtration is excreted as urine into the two ureter which are connected to urinary bladder where the ureter empties the urine [1,2].

The kidney plays an active role in the homeostasis of the human body through acid-base balance where many kinds of hormones such 
as antidiuretic hormone, aldosterone, angiotensin II and renin are active participants. As previously discussed kidney helps in reabsorption, filtration, secretion and excretion, around 180 liters of filtrate is generated by the kidney per day and after the reabsorption around 2 liters of urine is excreted [6].

\section{Why we need substitute for kidney i.e. Artificial Kidney?}

When the human kidneys are unable to perform their basic function the situation is termed as renal failure, this kind of situation may be for short term period (temporary failure) or for a very long term unspecified time period (permanent failure). After renal failure, the waste materials are deposited inside the blood which cause imbalance in homeostasis leading to the dysfunction of other organs including liver, heart that together hamper the body physiology. To solve this permanent problem the affected patient may require to replace the damaged kidney with donor kidney, but in case of unavailability of donor, then the ultimate solution for the patients is to undergo by the process of dialysis. Dialysis is a basic technical process where human blood is filtered in an artificial way just like the kidney and waste material such as salts, extra water, extra nutrients are removed [3,7].

\section{Some painful facts about dialysis}

It is a lifesaving process but it is very expensive treatment process. In a time period of one year near about $\$ 50,000$ cost is spent by the affected patient for the dialysis costs. Dialysis helps many patients to live normal life except the time period of dialysis which is painful time needed for treatments. Affected people those who depend on dialysis have to maintain a strict diet plan and they should limit their diets to minimize and reduce the harmful wastes. The lifestyle of the patients should be restricted in many manners [7].

Basically two types of dialysis are available such as hemodialysis by the help of a very costly machine and peritoneal dialysis which doesn't required any costly machine.

\section{Hemodialysis}

Hemodialysis is performed by an artificial machine called hemodialyzer which is otherwise known as artificial kidney, used for the removal of waste materials, toxic substances as well as fluid and extra chemicals from the human blood. For this process expert doctors have to deliver the blood to the machine and for this, they need to do surgery in arm/leg or may be by the fistula which is formed by the joining of artery and veins underneath the skin to create a blood vessel i.e. larger in size called as fistula.

It is a process of blood filtration by the help of hemodialysis machine, here the blood of the affected patient is passed through a dialysis venous catheter by means of a dialysis fluid solution into the machine which can filter the blood externally (outside the human body) and after filtration, the filtered blood is returned to the patient [8]. Maximum number of patients receives treatment of dialysis 3-4 times a week for 3-5 hours. Here the blood enters slowly inside the hemodialyzer and passes through tube bathed in dialysate solution and the tube having semipermeable membrane. During the process of filtration waste substances are diffused in the dialysate solution for removal and required substances were diffused into blood from the dialysate.

Risks of hemodialysis: There are some complications that may happen with hemodialysis such as poor blood flow, infection and blockage from clotting, cramping of muscle during the access of the patient's blood vessels. Sometime, a sudden drop in blood pressure is observed which can cause the affected patient to feel very weak, sick or dizzy. This lowering in blood pressure can cause sickness to the patient's stomach. The travel schedule for the patient should be restricted and should be wisely done as it has a great effect on their health status [9]. The quick reporting to the doctor and prompt treatment is necessary.

\section{Peritoneal dialysis}

In the process of peritoneal dialysis there is no requirement of costly machines. Here the blood of affected patient is cleaned inside the human body. The expert clinician places a catheter (which is a plastic tube) into the abdomen (belly) by means of an operation. Here the waste substances and unwanted fluid are removed away from the blood and turned into the dialysate and the blood remains inside the arteries and vein. During In the treatment time period the peritoneal cavity of the abdominal area is gradually filled with dialysate by means of catheter. Hence the unnecessary waste materials were removed from the blood as dialysate. The peritoneal dialysis is of two types: (i) Continuous ambulatory peritoneal dialysis and (ii) Continuous cycling peritoneal dialysis $[3,7,9]$.

\section{(i) Continuous ambulatory peritoneal dialysis}

Here the patient has to put about two liters of dialysate into his/her peritoneal cavity by means of a catheter four or five times a day. It should be done by the patient himself/herself, usually four or five times a day at home and/or at work. Then the dialysate removed back to the bag outside and have to be thrown away, each time patient required a new bag of dialysate.

\section{(ii) Continuous cycling peritoneal dialysis}

Here by means of a special machine termed as cycler the process can be done at home just as the similar process to be repeated as the above mentioned process of continuous ambulatory peritoneal dialysis except the number of cycles or exchanges to be done. Here each cycle usually lasts for half an hour to one hours and exchanges have to be done throughout that time period even during the night while the patient was in sleeping condition $[3,7,9]$.

Risk of peritoneal dialysis: One of the major and common problems with peritoneal dialysis is the development of a serious kind of abdominal infection called as peritonitis. This kind of infection can be treated with antibiotics by quick and prompt healthcare management. So it is advised to immediately report the expert clinician when such kind of issue is observed. Additionally, it should be reported to the doctor if the patients have fever. The quick reporting to the doctor and prompt treatment is necessary when the used dialysis solution has an unusual color or cloudiness and the area around the catheter is red or painful $[3,7,9]$.

\section{Conclusion}

The kidney is a major important organ of human being. Those patients having a crucial stage of kidney diseases have to be very strict about their lifestyle which includes their diet plans and exercise. The advice of clinician is needed in some serious cases where involvement of artificial kidney is required and under their supervision the patients has to be treated, because when kidney fails to provide its basic job then there is a necessity of artificial kidney for the waste removal and reabsorption purpose for a long lifespan. 
Citation: Pati P, Rathore SK (2018) The Necessity of Human Kidney and Artificial Kidneys for the Human Health. J Kidney 4: 166. doi:

Page 3 of 3

\section{References}

1. Standring S, Borley NR, Gray H (2008) Gray's anatomy: The anatomical basis of clinical practice, Churchill Livingstone/Elsevier.

2. Williams PL, Bannister LH, Berry MM (1995) Gray's Anatomy 38th Edn, Churchill Livingstone, London: 199-204.

3. Raghavendra, Mallikarjun, Vidya MJ (2013) Functions of kidney \& artificial kidneys. Int J Innovative Res Electrical Electronics Instrument Control Eng.

4. Boron WF (2004) Medical physiology: A cellular and molecular approach. Elsevier/Saunders.

5. Emamian SA, Nielsen MB, Pedersen JF, Ytte L (1993) Kidney dimensions at sonography: Correlation with age, sex and habitus in 665 adult volunteers. AJR Am J Roentgenol 160: 83-86.
6. Sembulingam K, Sembulingam P (2004) Essentials of medical physiology. Jaypee Publications.

7. Kidney failure: Choosing a treatment that's right for you (2001). NIH Publication.

8. Berman TA, Snyder S (2012) Kozier \& Erb's fundamentals of nursingconcepts, process and practice. 9th Edn, New Jersey: Pearson Education.

9. Wizemann V, Wabel P, Zaluska W, Chamney P, Moissl U, et al. (2009) The mortality risk in overhydration in hemodialysis patients. Nephrol Dial Transplant 24: 1574-1579. 